\title{
BMJ An exploratory study of cannabis open withdrawal among Indigenous Australian prison inmates: study protocol
}

\author{
Bernadette Rogerson, ${ }^{1}$ Jan Copeland, ${ }^{2}$ Petra Buttner, ${ }^{1}$ India Bohanna, ${ }^{1}$ \\ Yvonne Cadet-James, ${ }^{3}$ Zoltan Sarnyai, ${ }^{4}$ Alan R Clough ${ }^{1}$
}

To cite: Rogerson B, Copeland J, Buttner P, et al. An exploratory study of cannabis withdrawal among Indigenous Australian prison inmates: study protocol. BMJ Open 2013;3:e002951. doi:10.1136/bmjopen-2013002951

- Prepublication history and additional material for this paper is available online. To view these files please visit the journal online (http://dx.doi.org/10.1136/ bmjopen-2013-002951).

Received 26 March 2013 Accepted 5 April 2013

This final article is available for use under the terms of the Creative Commons Attribution Non-Commercial 2.0 Licence; see http://bmjopen.bmj.com

For numbered affiliations see end of article.

\section{Correspondence to:} Bernadette Rogerson; bernadette.rogerson1@jcu. edu.au.

\begin{abstract}
Introduction: Cannabis use and dependence is a serious health and criminal justice issue among incarcerated populations internationally. Upon abrupt, enforced cessation of cannabis, prisoners may suffer irritability and anger that can lead to threatening behaviour, intimidation, violence, sleep disturbances and self-harm. Cannabis withdrawal syndrome, proposed for inclusion in the Diagnostic and Statistical Manual of Mental Disorders in 2013, has not been examined in Indigenous populations. Owing to the exceptionally high rates of cannabis use in the community, high proportions of Australian Indigenous prisoners may suffer from withdrawal upon entry to custody.
\end{abstract}

Methods and analysis: 60 male and 60 female Indigenous prisoners (18-40 years) at a high risk of cannabis dependence will be recruited upon entry to custody. A pictorial representation of the standard Cannabis Withdrawal Scale will be tested for reliability and validity. Cortisol markers will be measured in saliva, as the indicators of onset and severity of cannabis withdrawal and psychological distress. The characteristics will be described as percentages and mean or median values with $95 \% \mathrm{Cl}$. Receiver operator curve analysis will determine an ideal cut-off of the Cannabis Withdrawal Scale and generalised estimating equations modelling will test changes over time. The acceptability and efficacy of proposed resources will be assessed qualitatively using thematic analysis.

Outcomes: A valid and reliable measure of cannabis withdrawal for use with Indigenous populations, the onset and time course of withdrawal symptoms in this population and the development of culturally acceptable resources and interventions to identify and manage cannabis withdrawal.

Ethics and dissemination: The project has been approved by the James Cook University Human Research Ethics Committee (approval number H4651).The results will be reported via peer reviewed publications, conference, seminar presentations and on-line media for national and international dissemination.

\section{INTRODUCTION}

Cannabis withdrawal is recognised as a consequence of cannabis dependence following

\section{ARTICLE SUMMARY}

Article focus

- The experience of cannabis withdrawal has not been the focus of any studies in Indigenous populations.

- This study aims to produce a valid and reliable measure of cannabis withdrawal for use with Indigenous populations and document the onset and time course of withdrawal symptoms.

- Studies with the potential for therapeutic outcome comprise a small proportion of published prisoner research.

Key messages

- A better understanding of cannabis withdrawal in custodial settings could assist in better risk management processes.

- Resources developed will assist new prison inmates to manage cannabis withdrawal and may assist communities to manage 'stressing out', widely observed and reported when cannabis is not available.

Strengths and limitations of this study

- There is a dearth of research pertaining to cannabis withdrawal in Indigenous populations, who are at a risk of cannabis-related harms owing to endemic levels of use with associated mental health issues.

- Custodial settings provide a controlled environment that addresses many of the methodological issues of previous studies.

- The main limitations of this study related to exploring illicit drug use is a stigmatised and sensitive topic, the study must fit within the tight schedules of a corrections environment and confounding variables such as stress owing to incarceration, withdrawal from tobacco and other substance use and pre-existing or undiagnosed mental health issues may impact the results.

the cessation of use. ${ }^{1}$ The symptoms include irritability, anger or aggression; nervousness or anxiety; insomnia; decreased appetite or weight loss; restlessness; depressed mood; and physical symptoms, for example, 
headache and sweating. Symptoms typically begin within $24 \mathrm{~h}$ of cessation, persisting for up to 28 days. $^{23}$

Cannabis withdrawal syndrome is proposed for inclusion in the next Diagnostic and Statistical Manual of Mental Disorders, fifth edition (DSM-5). ${ }^{4}$ Of particular clinical importance, withdrawal contributes to difficulties abstaining from cannabis ${ }^{5}$ and increases the likelihood of relapse in order to relieve withdrawal distress. ${ }^{6}{ }^{7}$ The proposed DSM-5 cannabis withdrawal syndrome criteria are informed by studies conducted mainly in treatmentseeking groups and adolescents, ${ }^{1}$ in whom higher rates of use and cannabis-related problems have been documented. $^{2}{ }^{45}$ No known published studies have specifically targeted ethnic minorities or marginalised subpopulations such as the First Nations and Indigenous peoples of North America or Australasia.

This study focuses on Indigenous Australians in one part of northern Australia, where the levels of cannabis use are very high. While cannabis use in Australia's general population may have been declining, ${ }^{8}$ the impacts of endemic cannabis use in remote Indigenous Australian communities are increasingly evident. ${ }^{9}$ Australian government surveys report past-year cannabis use for Indigenous Australians of $16 \%$ compared to $10 \%$ of the general population. ${ }^{8}$ Studies in remote communities in Australia's Arnhem Land, Northern Territory ${ }^{10}$ and Cape York, far north Queensland, ${ }^{11}$ have documented very high rates, up to $66 \%$ (in those aged 13-36 years), with a reported rapid rise in cannabis use in the late 1990s. ${ }^{10}$

Cannabis use is associated with criminal behaviours and incarceration. ${ }^{12}$ Unpublished pilot data were collected from 101 males incarcerated for 2 years or less in a far north Queensland correctional centre including $69 \%$ who were self-reported current cannabis users before incarceration: $70 \%$ recalling three or more symptoms of cannabis withdrawal during the week following their arrest. ${ }^{13}$ Participants also described 'stressing out' characterised by aggression, intimidation and anxiousness, contributing to disrupting family/relationships ${ }^{5}$ when cannabis was not available in their home community. 'Stressing out' may be a phenomenon similar to, if not the same as, cannabis withdrawal.

Indigenous Australians are incarcerated 15 times more frequently than the general population, ${ }^{14}$ with a rapidly increasing rate of incarceration for females of great concern. ${ }^{15}$ Higher rates of cannabis use are found in prison inmates and expectation of post-release drug use is a significant predictor of reincarceration. ${ }^{16}$ This project explores the nature and prevalence of the cannabis withdrawal syndrome and its measurement among individuals immediately following incarceration. The findings will enhance screening in prisons and similar environments and provide approaches to develop targeted interventions for cannabis withdrawal. The tightly controlled conditions of enforced abstinence in prison inmates close to entry into custody furnish a rare opportunity to understand cannabis withdrawal and the experience of 'stressing out' as well as an opportunity to address an unmet need for support in this highly marginalised and disadvantaged group.

The study objectives are to

1. Explore the experience of 'stressing out' in Indigenous Australians, identify culturallyspecific concepts of withdrawal, and use this information to inform the validation of a modified Cannabis Withdrawal Scale (I-CWS) for use with Indigenous Australian prison inmates.

2. Document onset, time course and severity of withdrawal symptoms in new Indigenous prison inmates who are dependent cannabis users, and compare this information with biological cortisol markers, taking into account recent alcohol and other drug use before arrest.

3. Using data from \#1 and \#2, and information from focus groups with prison inmates and prison staff, devise culturally acceptable, low-cost resources to assist in the management of cannabis withdrawal in controlled environments such as prisons and watch houses.

4. Disseminate resources to prison inmates and evaluate their acceptability and uptake.

\section{METHODS AND ANALYSIS}

\section{Study overview}

This study design is in line with the first and second phases in the schema of Campbell et al, ${ }^{17}$ for the design and implementation of complex interventions to improve health: 'modelling or identifying components of an intervention which are likely to work' and an 'exploratory trial of components'.

While Western views and assessment of mental health and ill health typically focus on personal experience, indigenous concepts of mental health are often more holistic and consider spirituality, environment, family and community in addition to personal experiences. ${ }^{18}$ Considering this, symptom assessment methodologies for cannabis withdrawal will be validated to ensure cultural relevance. Aspects of cultural equivalence will be explored systematically using both qualitative and quantitative measures. ${ }^{19}$

To accomplish the first objective, the validated CWS $^{2021}$ will be administered together with a semistructured interview to explore patterns and experiences of cannabis use. The themes in the data will assist to better understand symptoms and how they are experienced by inmates during initial incarceration when cannabis use is curtailed. Themes and symptoms will be considered by an expert reference group and cultural informants to develop a modified CWS, (I-CWS) for piloting.

For the second objective, new prisoners, within 1 week of incarceration, will undergo examination for symptomology and severity of cannabis withdrawal using a battery of scales, including the modified I-CWS measured on eight occasions over 28 days (figure 1 ).

The third and fourth objectives involve a series of focus groups comprised of prison inmates and prison staff. 


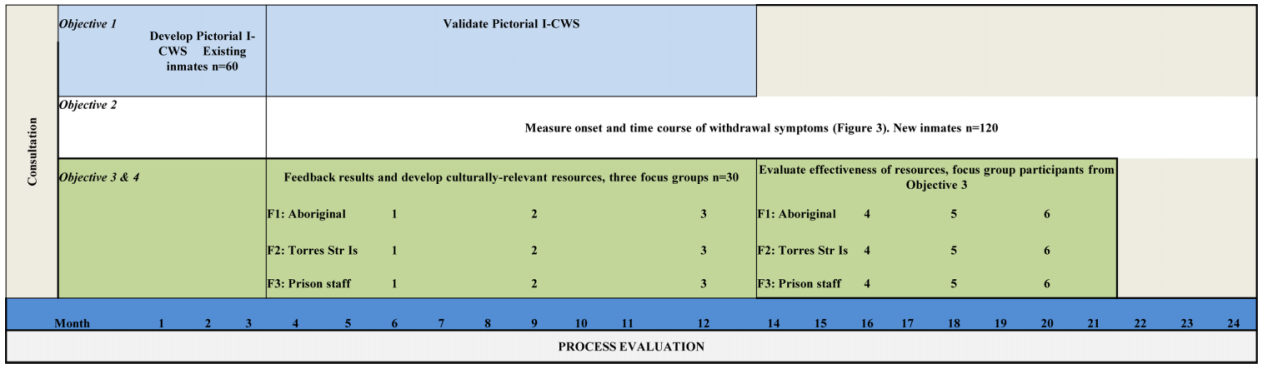

Figure 1 Assessments conducted over a 28-day period.

Semistructured interviews will be used to develop the I-CWS (Objective 1); the cannabis withdrawal assessments of new prison inmates (Objective 2) will be used to refine it during the first 12 months. The resource development phase (Objective 3) will overlap with interviews and assessments. The resource dissemination and evaluation phases (Objectives 3 and 4) will start by the 18th month and will be completed by the end of the 21st month. The design allows 24 months for interviews, assessments, focus groups, data analysis, research feedback and reporting (figure 2). A process evaluation will be undertaken over the course of the project.

\section{Setting}

Data will be collected using interviews with prisoners in three custodial localities in north Queensland-a watch house run by the Queensland Police Service (QPS) and two correctional centres run by Queensland Department of Corrections (QCS). North Queensland correctional centres report that $65-80 \%$ of prison inmates identify as Indigenous Australian, many of whom are from communities in Cape York and Torres Strait regions in far north Queensland.

Cape York covers 12 statistical regions, $\sim 128000 \mathrm{~km}^{2}$ (7.4\% of the total area of Queensland) with a population of approximately 7800 Indigenous Australians. ${ }^{22}$ The Torres Strait Islands is a cluster of over 250 islands $\sim 1380 \mathrm{~km}^{2} \quad(0.1 \%$ of the total area of Queensland) with a population of approximately 5900 Indigenous Australians. ${ }^{23}$
Specific methodological and ethical considerations arise in conducting research in a prison or detention environment. ${ }^{24}$ Involving prisoners as cultural informants to guide the recruitment process may assist to minimise the prospect that prisoners will feel coerced or that there is undue inducement or obligation to speak to a researcher. Additional measures must ensure that recruitment does not lead to any misapprehension of possible benefit including sentence reduction or help with parole. ${ }^{25}$

\section{Participant selection and recruitment}

Participant selection will occur in three stages outlined in figure 3. Firstly, to validate the I-CWS (Objective 1), participants will be prisoners who have been in custody for 2-24 months, be 18-40 years, Indigenous (Aboriginal and/or Torres Strait Islander) and will have tried cannabis at least once in the 12 months preceding incarceration to ensure variance in former levels of use and dependence. For Objective 2, participants will be new prisoners, 18-40 years, Indigenous, heavy cannabis users ( five or more days use on an average over the 3 months before prison), cannabis dependent and available to complete withdrawal symptom assessments on eight occasions over 28 days. Initial assessment will start at the QPS watch house and will be completed while in prison. To recognise cultural differences between Aboriginal and Torres Strait Islanders, Objective 3 will be achieved by inviting prison inmates to join one of two focus $(\mathrm{F})$ groups-Aboriginal (F1) and Torres Strait Islanders
Figure 2 Timeline to achieve Objectives 1-4.
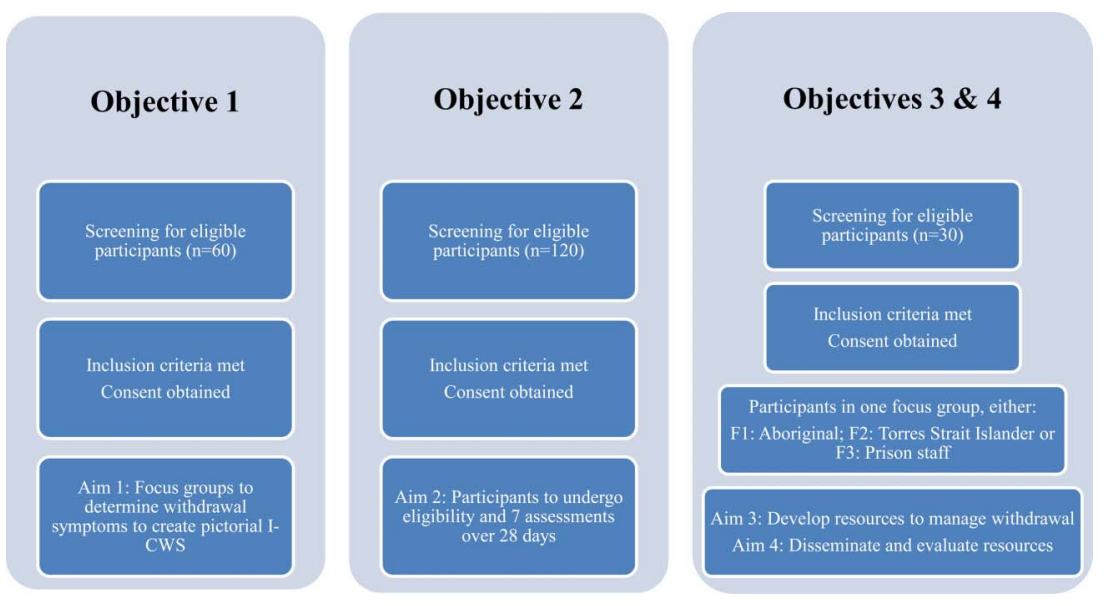
Figure 3 Participant selection for Objectives 1-4.

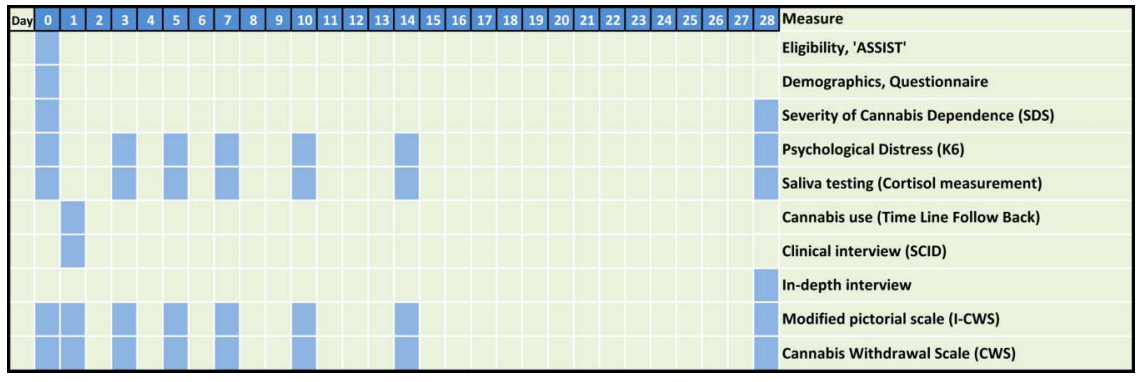

(F2). A third focus group (F3) will consist of QCS prison officers with at least 6 months service. Focus groups will guide the content and parameters of resource development to manage withdrawal, which will be disseminated before evaluating their utility and uptake (Objective 4).

Potential participants will be excluded if there are confounding health/mental health issues or if the participant is deemed unsuitable for interview by QCS staff. For each objective, the exclusion criteria are

Objectives 1, 3 and 4: those aged $<18$ and $>40$ years.

Objective 2: those aged $<18$ and $>40$ years, who are alcohol or other drug dependent (using National Health and Medical Research Council (NHMRC) guidelines ${ }^{26}$ and/or DSM dependence criteria and previously undergone any drug treatment, detoxification or rehabilitation.

Notices asking for volunteers to participate in the study will be placed within the correctional centres. To ascertain the eligibility for Objective 2, upon reception to the police watch house, QPS officers will notify researchers of those who are identified as Indigenous and who are aged between 18 and 40 years. Researchers will meet the detainee to provide an information sheet and explain the study purpose and design, voluntary participation and procedures for withdrawing from the study. Written informed consent will be obtained from those who wish to participate and a full eligibility assessment undertaken. Participants will be compensated $\$ 5$ for each assessment completed (total $\$ 40$ if all assessments completed) with the payment made to their institutional account. Paying prison inmates for participation is a matter of some controversy, but is common in criminological research. ${ }^{27}$ The initial battery of scales will be administered taking approximately $40 \mathrm{~min}$, with subsequent assessments requiring between 10 and 30 min (figure 1 ).

\section{Sample size calculation \\ Qualitative}

A purposive sample of 20 Indigenous prison inmates and 10 QCS prison officers will be invited to join one of three focus groups. Qualitative studies usually have smaller sample sizes of 10-20 deemed suitable to achieve data saturation. ${ }^{28}$

\section{Quantitative}

Pilot data ${ }^{13}$ provided estimates of the potential sample pool with $79 \%$ of prisoners being identified as
Indigenous, $69 \%$ as cannabis users and $84 \%$ who had experienced one or more withdrawal symptoms before incarceration. The sample pool will be 314 males (based on 750 new prison inmates estimated over study period) and 156 females (with an estimated 330 new prison inmates over study period).

A sample size of 60 men and 60 women is a practical and achievable target with the resources available. With the prevalence of withdrawal symptoms, expected from pilot data to be approximately $70 \%$, a precision of 0.264 can be achieved (95\% CI 0.568 to 0.812 ). These sample sizes provide study power in excess of $80 \%$ to detect differences between the eight repeated measures from baseline to 28 days (significance of 0.05 ) enabling a time series analysis of symptom score to be adjusted for three characteristics with one degree of freedom and still maintaining a stable model. ${ }^{29}$

\section{MEASURES/DATA COLLECTION Questionnaire adaptation}

An interactive and conversational approach, 'research yarning, ${ }^{30}$ is congruent with local and regional cultural processes. Questionnaires will be developed with focus groups and cultural informants using this approach to ensure appropriate wording/language, placement and cultural context. Qualitative data will be collected during this adaptation phase to ensure compatibility with cultural context and, once the questionnaires have been revised, a test-retest will be undertaken to ensure reliability.

\section{Information collection}

A series of open-ended questions will be used in the eligibility assessments to build rapport and to determine whether inclusion criteria are met. The timeline follow back method ${ }^{31}$ will be used at the eligibility interview to assess each participant's use of cannabis and other substances before incarceration, withdrawal symptoms, impact on family, finances and community, quitting intentions/attempts, understanding of withdrawal/'stressing out' and if any strategies were used to manage withdrawal.

Both qualitative and quantitative data will be collected during this phase. Participants will be asked their age, living arrangements before incarceration, education and work history. 


\section{Quantitative measures}

We will assess the participants at baseline and on days 1, $3,5,7,10,14$ and 28 following initial detention (figure 1). Alcohol, cannabis and other drug use will be measured using the Alcohol, Smoking and Substance Involvement Screening Test, ${ }^{32}$ with cannabis dependence measured at baseline and again at 28 days, using the Severity of Dependence Scale. ${ }^{33}$ As not all measures are validated for use with Indigenous prison inmates, structured clinical interviews ${ }^{34}$ will assess dependence, presence of mood episodes/disorders, psychotic and anxiety and adjustment disorders along with possible other drug dependence. To account for and monitor stresses experienced early in incarceration, psychological distress will be measured at baseline and subsequently on seven occasions using the modified Kessler 6 Psychological Distress Scale (K6), which has been used previously in Indigenous populations. ${ }^{35}$ Salivary cortisol levels, a non-intrusive objective marker of stress, ${ }^{36}$ will be assessed on the same days as psychological distress using the K6. The eight I-CWS assessments will be used to validate the CWS (figure 1).

This schedule is feasible within the routine of prison programming with 960 assessments (60 male and 60 female participants $\times 8$ occasions) to be conducted overall. At day 28, when withdrawal symptoms are likely to have subsided, ${ }^{37}$ a further assessment of dependence and an in-depth interview will also be conducted to explore qualitative experiences of withdrawal.

The research will be guided by experts in substance misuse and treatment including Indigenous cultural advisors, by cultural officers employed by QCS. Cultural informant groups, consisting of prison inmates at each site, will be formed through several group discussions. All groups will guide the development of the I-CWS to examine the validity of the symptoms and understanding of withdrawal and 'stressing out'. The groups will also provide input to the format of the I-CWS with regard to pictorial representation and the phrasing and wording of the symptom description.

\section{Process measures}

Resources aimed at enabling new prison inmates to recognise and manage symptoms of cannabis withdrawal will be developed and assessed within an action research framework, guided by the expert reference group, Indigenous advisors and cultural informants. These resources will be piloted with new prison inmates prior to dissemination for use by prison staff, as far as it is practicable. $^{38}$

Because of the national importance of reducing rates of incarceration in Indigenous people, ${ }^{39}$ the procedures used to engage QCS in embedding the resources into current practice will be described. We will record, via an implementation $\log$ completed by prison staff, the extent to which these resources are provided to, and used by, the prison inmate population.
Acceptability and feasibility will be determined by the administration of an additional questionnaire/interview at focus group meetings 4,5 and 6 (figure 2), the expert reference group, Indigenous advisors and cultural informants.

\section{PROPOSED INTERVENTIONS}

Treatments for cannabis withdrawal are only beginning to be investigated with no evidence-based interventions available for Indigenous populations. Currently, there is very little support for the treatment of cannabis-related problems for Indigenous populations, incarcerated or not. Managing withdrawal symptoms currently focuses on information provision and psychosocial interventions (eg, counselling and sleep management). ${ }^{4041}$

The complex relationship between drug use and criminal behaviour, with differences occurring between men and women, requires any treatment or intervention to target specific personal drug use and mental health histories. Factors common to drug use and crime (eg, sexual/ physical abuse, mental health problems, family problems) should be identified and treated concurrently. ${ }^{42}$

The focus groups will guide the development of the interventions and may range from brief interventions to stepped care models, based on psychosocial or social cognition therapies. Frameworks to be considered include psychoeducation, motivational enhancement, cognitive behaviour-based therapies, ${ }^{9}$ family and social emotional well-being $^{43}$ and/or social cognition training. ${ }^{44}$ It is intended that interventions will focus on continuing rehabilitation after release to assist with reintegration and management to reduce the risk relapse.

Intervention components will be developed in consultation with the focus groups to ensure cultural relevance and to align with best practice frameworks, so that they are practical, appropriate for the average level of cognitive function evident among prison inmates and address the identified needs for treatment. ${ }^{45}$

\section{ANALYSIS OF DATA}

\section{Qualitative analysis}

For Objective 1, themes described and symptoms experienced during initial incarceration and when cannabis use is curtailed will be documented. Using a simplified Delphi technique, already applied in Indigenous mental health research, ${ }^{46}$ themes and symptoms will be considered by the expert reference group and mapped against the CWS. Themes and symptoms will be provided to the expert reference group blinded and conducted in rounds until consensus is obtained.

To achieve the third and fourth objectives, qualitative information will be transcribed and analysed by two researchers. Sampling will continue until data are saturated with no new themes or categories emerging from the data. NVIVO software enables multilevel coding of text against a set of predetermined analytical categories. The research team will use an iterative process to 
understand the themes and key issues arising from the data, which will be coded independently with divergent views reconciled by a third researcher.

\section{Statistical analysis and data management}

To accomplish Objective 2, generalised estimating equations will be used to analyse Indigenous I-CWS scores over time. ${ }^{47}$ Modelling will test influences of time in abstinence from cannabis on total daily withdrawal scores (dependent variable) summed across all valid items in the Indigenous I-CWS. Demographic and other characteristics will be examined for their role in influencing scores over time.

Statistical analysis of quantitative data will be performed using IBM PASW SPSS software V.20.0 (IBM SPSS, Chicago, Illinois, USA) and analysis of qualitative data supported by QSR NVIVO V.10.

\section{LIMITATIONS}

Illicit drug use is a stigmatised and sensitive topic which may hinder recruitment and retention of participants. In addition, the study must fit within the tight schedules and local procedures at the three sites run by QPS and QCS. The study includes only two prison sites within Queensland and generalisations must be made with caution. However, this research is made possible by sound local relationships of trust with prison inmates with advice sought from the prison inmate reference group as cultural informants, enhancing its transferability. Protocols within the QPS watch house and QCS correctional centres will be adhered to at all times. The limited available resources prevent a larger scale multisite study.

There are risks associated with participation in this research which could implicate participants in further criminal charges if incriminating information is disclosed. Participants will be informed of this risk before involvement in the study and of the right to withdraw at any stage. A risk-management strategy ${ }^{24}$ proposes that matters of high risk and of intrinsic interest to law enforcement would be avoided in the study, that is, supply and distribution networks and participants' roles in them, matters related to other crimes not heard before a court or under investigation and/or matters related to any future planned crimes.

Confounding variables such as stress related to incarceration, withdrawal from tobacco and other substance use and pre-existing or undiagnosed mental health issues may impact the results.

\section{RELEVANCE}

There is a dearth of research pertaining to cannabis withdrawal in Indigenous populations, arguably the most at-risk populations for cannabis-related harms in Australia owing to endemic levels of use with associated mental health issues. Indigenous Australians are four to five times more likely than non-Indigenous Australians to be hospitalised for mental or behavioural disorders owing to the use of psychoactive substance. ${ }^{48}$ In remote communities in the Cape York region of far north Queensland, endemic cannabis has been linked to psychosis which is believed to play a role in the onset of illness in $61 \%$ of men and $37 \%$ of women (aged $\geq 15$ year).$^{49}$

Studies with the potential for therapeutic outcome comprise a small proportion of published prisoner research. ${ }^{39}$ In the USA, and mirrored in Australia, prisoners released to liberty are returning to their communities with a plethora of unaddressed health issues, particularly mental health and substance abuse problems. ${ }^{50}{ }^{51}$ Resources developed will assist new prison inmates to manage cannabis withdrawal and may assist communities to manage 'stressing out', widely observed and reported when cannabis is not available.

Of considerable importance, the results of this study will provide information about the onset, time-course and severity of withdrawal of populations in a nontreatment study population, the focus of few cannabis withdrawal studies. Custodial settings provide a controlled environment that addresses many of the methodological issues of previous studies where participant recruitment, drop out and abstinence has been problematic.

The strength of this research team is its capacity to translate the practical outcomes of the study to the development of sustainable, easily delivered resources to better manage mental health, withdrawal and cannabis-related issues in newly incarcerated Indigenous prison inmates.

\section{ETHICS AND DISSEMINATION}

The James Cook University (JCU) Human Research Ethics Committee has approved the proposal for the study H4651, as well as related information sheets and consent forms summarising the research and roles of potential participants. All project data will be deidentified by removing the statements identifying participants prior to their use in published materials. All questionnaire, interview and focus group data will be stored securely in password-protected folders accessible only to the research team. In accordance with Australia's NHMRC guidelines, ${ }^{52}$ all project data will be retained for a minimum of 7 years. Participants will be provided with contact details of the research team to enable them to address any potential queries or concerns. Any complaints will be systematically recorded and acted upon in accordance with JCU guidelines. Participants will be informed of their right to access their own results, and the overall results of the research, in accordance with NHMRC guidelines. ${ }^{51}$ Journal articles developed from the research will be sent to all project partners for dissemination to participants, and deidentified results provided in progress reports to QCS and QPS. Conference and seminars will be presented to Australian and international healthcare stakeholders, researchers and academics. 
Author affiliations

${ }^{1}$ School of Public Health, Tropical Medicine and Rehabilitation Sciences, James Cook University, Cairns and Townsville, Queensland, Australia ${ }^{2}$ National Cannabis Prevention \& Information Centre, UNSW Medicine, Sydney, New South Wales, Australia

${ }^{3}$ School of Indigenous Australian Studies, James Cook University, Townsville, Queensland, Australia

${ }^{4}$ School of Veterinary and Biomedical Sciences, James Cook University, Townsville, Queensland, Australia

Acknowledgements We thank Queensland Police Service and Queensland Corrective Services for granting research approvals to conduct the study. We thank Professor Ernest Hunter for his contribution to the original study design and Associate Investigators Ms Kimina Andersen, Professor Wayne Hall, Dr Edward Heffernan, Dr Garry Kidd, Mr Darryl Joseph, Mr Greg Kingston and Research Officers Liana Schnierer and Michelle Coleman.

Contributors BR and ARC conceived the original concept of the study. ARC led the design of the original funded study protocol. BR provided pilot data for original funded study protocol. BR, IB, JC, PB, YCJ designed the original funded study protocol. BR obtained Ethics approval and wrote the first draft of the manuscript. All authors contributed to drafts of the manuscript and approved the final version for publication.

Funding This work is supported by the National Medical Health Research Council (Australia) grant number 1020514. ARC is the Chief Investigator and holder of the NHMRC funding, JC, PB and YCJ are co-Chief Investigators and ZS is an Associate Investigator.

Competing interests Study components form part of Doctor of Philosophy degree for author BR.

Ethics approval The study was approved by James Cook University Human Research Ethics Committee (H4651).

Provenance and peer review Not commissioned; peer reviewed for ethical and funding approval prior to submission. Formal ethical approval from James Cook University and funding from NHMRC (JULIET project).

\section{REFERENCES}

1. Budney AJ, Moore BA. Development and consequences of cannabis dependence. J Clin Pharmacol 2002;42(Suppl 11):S28-33.

2. Hall W, Degenhardt L, Lynskey M. The health and psychological effects of cannabis use. Monograph no. 44. 2nd edn. Canberra, Australia: Commonwealth Department of Health and Ageing, 2001

3. Kouri E, Pope Jr H. Abstinence symptoms during withdrawal from chronic marijuana use. Exp Clin Psychopharmacol 2000;8:483-92.

4. R 10 Cannabis Withdrawal. American Psychiatric Association, DSM-5 Development. http://www.dsm5.org/ProposedRevision/ Pages/proposedrevision.aspx?rid=430 (accessed 17 Aug 2012).

5. Budney A, Radonovich K, Higgins S, et al. Adults seeking treatment for marijuana dependence: a comparison with cocaine-dependent treatment seekers. Exp Clin Psychopharmacol 1998;6:419-26.

6. Budney AJ, Novy PL, Hughes JR. Marijuana withdrawal among adults seeking treatment for marijuana dependence. Addiction 1999;94:1311-22.

7. Stephens RS, Roffman RA, Simpson EE. Adult marijuana users seeking treatment. J Consult Clinical Psychol 1993;61:1100-4.

8. Australian Institute of Health and Welfare. 2010 National Drug Strategy Household Survey report. AlHW cat. no. PHE 1452011. http://www.aihw.gov.au/publication-detail/?id=32212254712 (accessed 17 Aug 2012).

9. Lee KSK, Clough AR, Jaragba M, et al. Cannabis in the 'top end'. Of Subst 2008;6:18-9.

10. Lee KS, Conigrave KM, Clough AR, et al. Five-year longitudinal study of cannabis users in three remote Aboriginal communities in Arnhem Land, Northern Territory, Australia. Drug Alcohol Rev 2009;28:623-30.

11. Bohanna I, Clough A. Cannabis use in Cape York Indigenous communities: high prevalence, mental health impacts and the desire to quit. Drug Alcohol Rev 2011;31:580-4.

12. Wilkins $C$, Sweetsur $P$. The association between spending on methamphetamine/amphetamine and cannabis for personal use and earnings from acquisitive crime among police detainees in New Zealand. Addiction 2011;106:789-97.
13. Rogerson B. Cannabis use, dependence and withdrawal [Honours Thesis]. Cairns, Australia: James Cook University, 2010.

14. Australian Bureau of Statistics. Prisoners in Australia. ABS cat. no. 4517.0 2012. http://www.abs.gov.au (accessed 17 Feb 2013).

15. Australian Institute of Health and Welfare and Australian Bureau of Statistics, The Health and Welfare of Australia's Aboriginal and Torres Strait Islander Peoples, 2008. ABS cat no. 4704.02008 http://www.abs.gov.au (accessed 17 Aug 2012).

16. Payne J, Macgregor S, McDonald H. Research into Practice Brief 7: Prevalence and issues relating to cannabis use among prison inmates: Key findings from Australian research since 20012013. http://ncpic.org.au/ncpic/publications/aic-bulletins/article/aicresearch-into-practice-brief-7-prevalence-and-issues-relating-tocannabis-use-among-prison-inmates-key-findings-from-australianresearch-since-2001 (accessed 3 Mar 2013)

17. Campbell M, Fitzpatrick R, Haines A, et al Framework for design and evaluation of complex interventions to improve health. $\mathrm{Br} \mathrm{Med} \mathrm{J}$ 2000;321:694-6.

18. Dingwall KM, Cairney S. Psychological and cognitive assessment of Indigenous Australians. Aust NZ J Psychiatry 2010;44:20-30.

19. Esler DM, Johnston F, Thomas D. The acceptability of a depression screening tool in an urban, aboriginal community controlled health service. Aust NZ J Public Health 2007;31:259-63.

20. Allsop DJ, Copeland J, Norberg MM, et al. Quantifying the clinical significance of cannabis withdrawal. PLOS ONE 2012;7:e44864.

21. Allsop DJ, Norberg MM, Copeland J, et al. The cannabis withdrawal scale development: patterns and predictors of cannabis withdrawal and distress. Drug Alcohol Depend 2011;119:123-9.

22. Queensland Treasury and Trade Government Statistician. Queensland Regional Profile for Cape York Region. 2012. http://statistics.oesr.qld.gov.au/qld-regional-profiles (accessed 20 Feb 2013)

23. Queensland Treasury and Trade Government Statistician. Queensland Regional Profile for Torres Strait Region. 2012. http://statistics.oesr.qld. gov.au/qld-regional-profiles (accessed 20 Feb 2013).

24. Schlosser JA. Issues in interviewing inmates: navigating the methodological landmines of prison research. Qual Inq2008;14:1500-25.

25. Copes H, Hochstetler A, Brown A. Inmates' perceptions of the benefits and harm of prison interviews. Field Methods 2012;00:1-15

26. National Health and Medical Research Council. Australian guidelines to reduce health risks from drinking alcohol. Canberra, Australia: NHMRC, 2009: 1-179.

27. Hanson RK, Letourneau EJ, Olver ME, et al. Incentives for offender research participation are both ethical and practical. Crimi Justice Behav 2012;39:1391-404.

28. Guest G, Bunce A, Johnson L. How many interviews are enough? An experiment with data saturation and variability. Field Methods 2006;18:59-82.

29. Feinstein A. Multivariable analysis: an introduction. New Haven, CT: Yale University Press, 1996:241-7.

30. Moreton-Robinson AM, Walter M. Indigenous methodologies in social research. In: Walter M.ed Social research methods. Melbourne, Australia: Oxford University Press, 2009:1-18.

31. Sobell LC, Sobell MB. Alcohol timeline followback users' manual. Toronto, Canada: Addiction Research Foundation, 1995.

32. Ali RL, Awwad E, Babor T, et al. The Alcohol, Smoking and Substance Involvement Screening Test (ASSIST): development, reliability and feasibility. Addiction 2002;97:1183-94.

33. Gossop M, Best D, Marsden J, et al. Test-retest reliability of the severity of dependence scale. Addiction 1997;92:353.

34. First MB, Gibbon M, Spitzer RL, et al. User's guide for the structured clinical interview for DSM-IV xis II personality disorders. Washington, DC: American Psychiatric Press, 1997.

35. Haswell MR, Kavanagh D, Tsey K, et al. Psychometric validation of the growth and empowerment measure (GEM) applied with indigenous Australians. Aust N Z J Psychiatry 2010;44:791-9.

36. Schmitt LH, Harrison GA, Spargo RM. Variation in epinephrine and cortisol excretion rates associated with behavior in an Australian aboriginal community. Am J Phys Anthropol 1998;106:249-53.

37. Budney AJ, Moore BA, Vandrey RG, et al. The time course and significance of cannabis withdrawal. J Abnorm Psychol 2003;112:393-402.

38. Carroll C, Patterson M, Wood S, et al. A conceptual framework for implementation fidelity. Implement Sci 2007;2:40.

39. Australian Parliament, House of Representatives Standing Committee on Aboriginal and Torres Strait Islander Affairs, Doing time-Time for doing. Indigenous youth in the criminal justice system. Canberra, Australia: The Parliament of the Commonwealth of Australia, 2011:1-378.

40. Budney AJ, Hughes JR. The cannabis withdrawal syndrome. Curr Opin Psychiatry 2006;19:233-8. 
41. Copeland J, Frewen A, Elkins K. Management of cannabis use disorder and related issues: a clinician's guide 2009. http://ncpic.org $\mathrm{au} / \mathrm{ncpic} /$ news/ncpic-news/article/management-of-cannabis-usedisorder-and-related-issues-a-clinicians-guide (accessed 17 Aug 2012)

42. Johnson $\mathrm{H}$. Drugs and crime: a study of incarcerated female offenders. Research and Public Policy Series no 63. Canberra, Australia: Australian Institute of Criminology, 2004:93-103.

43. Haswell M, Hunter E, Wargent R, et al. Protocols for the delivery of social and emotional wellbeing and mental health services in Indigenous communities: guidelines for health workers, clinicians, consumers and carers, 1st edn. Cairns: Australian Integrated Mental Health Initiative, Queensland Health, 2009. http://www.uq.edu.au/ nqhepu/documents/protocols.pdf (accessed 18 Mar 2013).

44. Roberts DL, Penn DL, Labate D, et al. Transportability and feasibility of Social Cognition And Interaction Training (SCIT) in community settings. Behav Cog Psychother 2010;38:35-47.

45. Westerman TG, Wettinger M. Working with Aboriginal People. Psychologically Speaking, Perth, Western Australian Psychological Society_Psychological Assessment of Aboriginal People. 1998. http://www/indigenouspsychservices.com.au/Publications (accessed 11 Mar 2013)

46. Hart LM, Jorm AF, Kanowski LG, et al. Mental health first aid for Indigenous Australians: Using Delphi consensus studies to develop guidelines for culturally appropriate responses to mental health problems. BMC Psychiatry 2009;9:47.

47. Lee JH, Herzog TA, Meade CD, et al. The use of GEE for analyzing longitudinal binomial data: a primer using data from a tobacco intervention. Addict Behav 2007;32:187-93.

48. Australian Institute of Health and Welfare. 2006 Aboriginal and Torres Strait Islander Health Performance Framework. AlHW cat. no. IHW 20 2007. http://www.aihw.gov.au/publication-detail/? id=6442467995 (accessed 17 Aug 2012).

49. Hunter E, Gynther B, Anderson C, et al. Psychosis in Aboriginal and Torres Strait Islander populations of Cape York and the Torres Strait. Med J Aust 2011;196:133.

50. Davis LM, Pacchiana S. Health profile of the state prison population and returning offenders: public health challenges. J Correct Health Care 2004; 10:303-31.

51. Borzycki M, Baldry E. Promoting integration: the provision of prisoner post-release services. Trends \& issues in crime and criminal justice no. 262. Canberra, Australia: Australian Government, Australian Institute of Criminology, 2003.

52. National Health and Medical Research Council, Australian Research Council, Australian Vice-Chancellors' Committee. National statement on ethical conduct in human research. Canberra, Australia: Australian Government, 2007. Updated 2009. http://www.nhmrc.gov.au/_files_ nhmrc/publications/attachments/e72.pdf (accessed $17 \mathrm{Feb} 2013$ ). 